\title{
Surgical Results of Anderson-Hynes Dismembered Pyeloplasty Without Internal Drainage in Adults With Ureteroplevic Junction Obstruction
}

\author{
Aliasghar Yarmohamadi ${ }^{1}$; Parisa Saeedi ${ }^{1,} ;$;mir Hoghabrosadat ${ }^{1}$ \\ ${ }^{1}$ Department of Urology, Mashhad University of Medical Sciences, Mashhad, IR Iran \\ *Corresponding author: Parisa Saeedi, Department of Urology, Mashhad University of Medical Sciences, Mashhad, IR Iran. Tel: +98-9155161787, Fax: +98-5138820417, E-mail: saeedip@mums.ac.ir
}

Received: July 13, 2014; Revised: September 8, 2014; Accepted: November 27, 2014

\begin{abstract}
Background:Anderson-Hynes dismembered pyeloplasty is the gold standard therapeutic approach to ureteropelvic junction obstruction (UPJO). Use of a drainage method to protect the suture line from leakage is a matter of controversy.

Objectives: We have compared the surgical outcome of Anderson-Hynes dismembered pyeloplasty for UPJO repair, with or without internal stenting.

Patients and Methods: Eighty-two patients with UPJO were evaluated from 1996 to 2002 . Complicated or emergent cases were excluded. Classic standard dismembered pyeloplasty was performed. Internal drainage, with a double $\mathrm{j}$ catheter, was performed in several patients, randomly. Another drain was also placed in the retroperitoneal space. The follow-up of patients was planned weekly, with patient visits and urine analysis and intravenous pyelography (IVP) and diethylene-triamine-pentaacetate (DTPA) scan after one month.

Results: The study group consisted of 51 male and 31 female patients, who were mostly in the age range of 20 - 40 years. Comparing the two techniques of pyeloplasty with or without internal drainage, there was no significant difference between groups regarding extravasation and anastomosis complications, such as leakage, stenosis, urinoma formation or evidence of obstruction on postoperative IVP or DTPA scan. However, a higher incidence of catheter related urinary symptoms and flank pain was reported among those with internal stent. Conclusions: Pyeloplasty, with adequate spatulation, hemostasis and a watertight anastomosis, represents the mainstay of successful pyeloplasty and there may be no significant benefit for urethral stenting, especially in non-complicated cases.
\end{abstract}

Keywords: Urology; Surgery; Drainage

\section{Background}

Anderson-Hynes dismembered pyeloplasty was first introduced in 1949 for the treatment of obstructed retrocaval ureter. However, it is still the gold standard therapeutic approach to ureteropelvic junction obstruction (UPJO) (1). The success rate of this procedure is estimated as high as $90 \%$ (2). The mainstay of this surgical intervention is to respect the stenotic part and the enlarged part of the ureter, while performing repositioning of ureter and ureteropelvic anastomosis (3). Use of a drainage method to protect the suture line from leakage is a matter of controversy (4), as retrograde and antegrade double J(DJ) stenting is used as a splint cross the anastomosis (5). Currently, DJ internal drainage is widely used by those surgeons who advocate the drainage methods. Nevertheless, several stent related symptoms are reported in these cases and readmission of patients is necessary for catheter removal, which may impose extra charges to these patients (6). Similar recent researches support stentless open pyeloplasty in uncomplicated cases (7).

\section{Objectives}

The objective of this study was to compare the surgical outcome of Anderson-Hynes dismembered pyeloplasty for UPJO repair with or without internal stenting.

\section{Patients and Methods}

Eighty two patients with UPJO were evaluated from 1996 to 2002 in the academic center of Mashhad University of Medical Sciences, Mashhad, Iran. The study population comprised of 51 male and 31 female patients in the age range of 17-49 years. Patients were divided into two groups randomly, according to stent usage. The UPJO diagnosis was made on the basis of patient reported symptoms, sonographic findings and intravenous pyelography (IVP) and diethylene-triamine-pentaacetate (DTPA) scan results. Those cases with renal failure, history of previous UPJO repair or requiring emergent drainage because of renal stone or infection were all excluded. Surgical intervention was performed through a flank incision via an intraperitoneal and extraperitoneal approach. Classic standard dismembered pyeloplasty was performed. After completion of the posterior suture line, a DJ catheter was paced in the internal drainage group (randomly selected) while in other stentless cases, a temporary feeding tube was inserted for secure anastomosis, which was later removed. Anastomosis was performed using 4-0 chromic separate sutures in a water tight fashion. Pyeloplasty was performed by continuous interlock sutures where needed. A drain was

Copyright (C) 2015, Nephrology and Urology Research Center. This is an open-access article distributed under the terms of the Creative Commons Attribution-NonCommercial 4.0 International License (http://creativecommons.org/licenses/by-nc/4.0/) which permits copy and redistribute the material just in noncommercial usages, provided the original work is properly cited. 
also placed in the retroperitoneal space and fallow-up of patients was planned weekly. Drain output was assessed and it was extracted when drainage became less than 50 cc per day. All cases were also assessed for fever and urinary tract infection symptoms. Urine analysis was done and oral antibiotics were continued for at least 5 days after discharge. The IVP was performed after 4 weeks in all patients and the renal function and probable extravasation and structural anomalies were evaluated. Those patients with internal stent were admitted again after 1 month to remove the stent if no complication had occurred. A 3 day course of oral antibiotic therapy was also started at the time of stent removal. Statistical analysis was performed with the SPSS software Ver. 11.5 (SPSS Inc., Chicago, IL, USA) using T standard and Chi-Square tests. A P $<0.05$ was considered significant.

\section{Results}

Double stenting was used in 30 cases and pyeloplasty without internal drainage was performed in 52 patients. Both groups were similar in age and sex distribution. The most common symptom was severe flank pain, which was present in $69 \%$ of patients. History of urinary tract infection was detected in $47 \%$ and 51\% reported associated gastrointestinal symptoms. Pyuria was seen in 58\% and only $36 \%$ of patients had positive urine culture, with Escherichia coli (E. coli) as the most common pathogen (60\%). The preoperative sonographic evaluation and IVP results are shown in Table 1. Intraoperative assessments showed ureteropelvic junction segmental atresia in 45 cases and aberrant vessels in 25 patients. The postoperative course was compared in two groups of patients, with and without internal drainage (Table 2). Among patients with extravasation in DTPA scan

\begin{tabular}{|c|c|c|}
\hline Imaging Method & Right Kidney & Left Kidney \\
\hline \multicolumn{3}{|l|}{ Sonography } \\
\hline Severe hydronephrosis & $28(34.6)$ & $35(43.2)$ \\
\hline Moderate hydronephrosis & $8(9.9)$ & $9(11.1)$ \\
\hline Mild hydronephrosis & $6(7.4)$ & $3(3.7)$ \\
\hline Without hydronephrosis & $39(48)$ & $34(42)$ \\
\hline \multicolumn{3}{|l|}{ IVP } \\
\hline No secretion & $6(7.3)$ & $9(11)$ \\
\hline Delayed secretion & $33(40.2)$ & $38(46.3)$ \\
\hline Normal secretion & $43(52.4)$ & $35(42.7)$ \\
\hline
\end{tabular}

a Abbreviation: IVP, intravenous pyelography

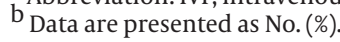

\begin{tabular}{|c|c|c|c|}
\hline Post-Operative Course & With Stent $(\mathbf{n}=\mathbf{3 0})$ & Without Stent $(n=52)$ & PValue \\
\hline \multicolumn{4}{|l|}{ Drain removal } \\
\hline In 48 hours & $12(40)$ & $17(32.7)$ & 0.583 \\
\hline After 2 days & $18(60)$ & $34(65.4)$ & 0.632 \\
\hline Mean hospital stay, d & $36 \pm 1.74$ & $3.4 \pm 1.30$ & 0.841 \\
\hline \multicolumn{4}{|l|}{ Urine analysis ${ }^{C}$} \\
\hline Hematuria & $19 / 27$ & $32 / 32$ & 0.001 \\
\hline Pyuria & $12 / 27$ & $20 / 32$ & 0.008 \\
\hline \multicolumn{4}{|c|}{ Post-operative IVP (one month later) } \\
\hline Extravasation & $2(6.7)$ & $8(15.3)$ & 0.209 \\
\hline Delayed secretion & $19(63.3)$ & $25(48.1)$ & 0.124 \\
\hline Visible ureter & $18(60)$ & $41(78.8)$ & 0.633 \\
\hline \multicolumn{4}{|l|}{ DTPA scan $^{\mathrm{d}}$} \\
\hline Partial obstruction & $8 / 20$ & $13 / 44$ & 0.716 \\
\hline Urinary symptoms & $24(80)$ & $2(3.8)$ & 0.005 \\
\hline Urinoma & $0(0)$ & $1(1.9)$ & 0.324 \\
\hline \multicolumn{4}{|l|}{ Recurrence of stenosis } \\
\hline reoperation & $0(0)$ & $1(1.9)$ & 0.324 \\
\hline Stent remnants & $2(6.7)$ & $0(0)$ & 0.041 \\
\hline Flank pain at voiding & $10(33.3)$ & $0(0)$ & 0.001 \\
\hline
\end{tabular}

\footnotetext{
a Abbreviations: IVP, intravenous pyelography; DTPA, diethylene-triamine-pentaacetate.

$\mathrm{b}$ Data are presented as No. (\%).

${ }^{C}$ Urine analysis was done in 27 patients of the stent group and 32 cases of the stent free group.

$\mathrm{d}$ DTPA scan was done in 20 patients of stent group and 32 cases of stent free group.
} 
in the stent free group, retrograde DJ stent was inserted in four cases that had unacceptable drainage due to inflammation. One patient had distal ureter obstruction because of distal ureter stone, that was treated by Trans-ureteral lithotripsy (TULP) and stent support. Three other cases had no anatomical abnormality, and they were followed and extravasation resolved spontaneously at control IVP after one month. Removal of DJ stent was planned in one month after operation. However, eight cases missed the time and late stent removal was accompanied with several complications, such as stone formation on the tip of catheter in the bladder of a patient that needed TULP before stent removal.

\section{Discussion}

The UPJO is a common anomaly, especially encountered in pediatric urology, which is traditionally treated by open pyeloplasty and the most common method is the Anderson-Hynes dismembered pyeloplasty, performed via a retroperitoneal approach by a flank incision. According to the original approach, insertion of stent in not necessary in this procedure except for complicated cases or doubtful anastomosis (1). In recent decades and with the introduction of new stents, such as the DJ stents, internal drainage has become popular as a result of the means of anastomosis support, lowering the risk of extravasation and urinoma formation, ensuring urinary drainage, maintain ureteral caliber and preventing the obstruction due to anastomotic site edema (7). Recently, and especially by promotion of minimally invasive methods and laparoscopic repair of UPJO, an ongoing debate is seen regarding the pros and cons of internal drainage in UPJO repair. Stents associated symptoms, such as dysuria, frequency, flank pain, hematuria, are commonly reported, even with short term placemat of urethral catheters, that may be so severe in selected cases that they even interfere with daily activities and impair the quality of life (8). Joshi et al. reported these bothering urinary complains in $78 \%$ of cases with ureteral stent (9). Among these patients, $80 \%$ experienced voiding or flank pain, 32\% sexual impairment and 58\% the negative effects on normal life (9). Potential catheter related complications include catheter dislocation or migration that may cause stent dysfunction and even obstructive uropathy, retained fragments, stone formation on the foreign body, exposure of upper system to high pressure during voiding and an artificial reflux uropathy, flank pain and an increased rate of catheter related urinary infections $(7,10,11)$. The need for readmission and general anesthesia occurs, especially in children. Several attempts have been done to prevent readmission and repeated general anesthesia for catheter removal, and unfortunately they failed $(12,13)$. In our study comparing two techniques of pyeloplasty with or without internal drainage, there was no significant difference between groups, concerning extravasation and anastomosis complications, such as leakage, stenosis, urinoma formation, evidence of obstruction at postoperative IVP or DTPA scan. However, a significant incidence of catheter related symptoms, such as irritative urinary symptoms and flank pain was reported. Similar studies, such as Kumar (8) or Nguyen et al. (14), also reported almost the same results. In a case series of non-intubated pyeloplasty by Nguyen et al. ureteral stent was not recommended for uncomplicated cases (14). Kumar et al. also concluded that stentless pyeloplasty may be a feasible option in adults (8).

In conclusion, pyeloplasty with proper spatulation, hemostasis and a watertight anastomosis is the mainstay of a successful pyeloplasty and there may be no significant benefit for urethral stenting, especially in non-complicated cases.

\section{References}

1. Anderson JC, Hynes W. Retrocaval ureter; a case diagnosed preoperatively and treated successfully by a plastic operation. $\mathrm{Br} J$ Urol.1949;21(3):209-14.

2. Iwamura M, Soh S, Irie A, Kadowaki K, Matsusita Y, Fujioka T, et al. Laparoscopic pyeloplasty for ureteropelvic junction obstruction: outcome of initial 12 procedures. Int J Urol. 2004;11(7):44955.

3. Symons SJ, Bhirud PS, Jain V, Shetty AS, Desai MR. Laparoscopic pyeloplasty: our new gold standard.J Endourol. 2009;23(3):463-7.

4. Mandhani A, Goel S, Bhandari M. Is antegrade stenting superior to retrograde stenting in laparoscopic pyeloplasty? J Urol. 2004;171(4):1440-2.

5. Mandhani A, Kumar D, Kumar A, Dubey D, Kapoor R. Steps to reduce operative time in laparoscopic dismembered pyeloplasty for moderate to large renal pelvis. Urology. 2005;66(5):981-4.

6. Shalhav AL, Mikhail AA, Orvieto MA, Gofrit ON, Gerber GS, Zorn KC. Adult stentless laparoscopic pyeloplasty. JSLS. 2007;11(1):8-13.

7. Smith KE, Holmes N, Lieb JI, Mandell J, Baskin LS, Kogan BA, et al. Stented versus nonstented pediatric pyeloplasty: a modern series and review of the literature. JUrol.2002;168(3):1127-30.

8. Kumar V, Mandhani A. Laparoscopic stentless pyeloplasty: an early experience. Indian J Urol. 2010;26(1):50-5.

9. Joshi HB, Stainthorpe A, MacDonagh RP, Keeley FJ, Timoney AG, Barry MJ. Indwelling ureteral stents: evaluation of symptoms, quality of life and utility. J Urol. 2003;169(3):1065-9.

10. Richter S, Ringel A, Shalev M, Nissenkorn I. The indwelling ureteric stent: a 'friendly' procedure with unfriendly high morbidity. BJU Int. 2000;85(4):408-11.

11. Bryant RJ, Craig E, Oakley N. Laparoscopic pyeloplasty: the retroperitoneal approach is suitable for establishing a de novo practice. J Postgrad Med. 2008;54(4):263-7.

12. Macaluso JJ, Deutsch JS, Goodman JR, Appell RA, Prats LJ, Wahl P. The use of the Magnetip double-J ureteral stent in urological practice. JUrol.1989;142(3):701-3.

13. Taveres A, Manaboriboon N, Lorenzo AJ, Farhat WA. Insertion of an internal-external nephroureteral stent during pediatric laparoscopic pyeloplasty: description of the technique. Urology. 2008;71(6):1199-202.

14. Nguyen DH, Aliabadi H, Ercole CJ, Gonzalez R. Nonintubated Anderson-Hynes repair of ureteropelvic junction obstruction in 60 patients. J Urol. 1989;142(3):704-6. 